\title{
DW-MRI in assessment of the hypoxic fraction, interstitial fluid pressure, and metastatic propensity of melanoma xenografts
}

\author{
Tord Hompland, Christine Ellingsen, Kanthi Galappathi and Einar K Rofstad ${ }^{*}$
}

\begin{abstract}
Background: Cancer patients with primary tumors showing extensive hypoxia and highly elevated interstitial fluid pressure (IFP) have poor prognosis. The potential of diffusion-weighted magnetic resonance imaging (DW-MRI) in assessing the hypoxic fraction, IFP, and metastatic propensity of tumors was investigated in this study.

Methods: A-07 and R-18 melanoma xenografts were used as general models of human cancer. DW-MRI was performed at 1.5 T, and maps of the apparent diffusion coefficient (ADC) were produced with in-house-made software developed in Matlab. Pimonidazole was used as a hypoxia marker. Tumor cell density and hypoxic fraction were assessed by quantitative analysis of histological sections. IFP was measured with a Millar catheter. Metastatic propensity was determined by examining tumor-bearing mice for pulmonary micrometastases post mortem.

Results: ADC decreased with increasing tumor cell density, independent of whether the A-07 and R-18 data were analyzed separately or together. In the A-07 line, ADC decreased with increasing hypoxic fraction and increasing IFP and was lower in metastatic than in nonmetastatic tumors, and in the R-18 line, ADC decreased with increasing hypoxic fraction. There was a strong inverse correlation between ADC and hypoxic fraction as well as between ADC and IFP across the two tumor lines, primarily because low ADC as well as high hypoxic fraction and high IFP were associated with high cell density.

Conclusion: Low ADC is a potentially useful biomarker of poor prognosis in cancer, since low ADC is mainly a consequence of high cell density, and high cell density may lead to increased hypoxia and interstitial hypertension and, therefore, increased microenvironment-associated metastasis.
\end{abstract}

Keywords: DW-MRI, Hypoxia, Interstitial fluid pressure, Metastasis, Cell density

\section{Background}

Diffusion-weighted magnetic resonance imaging (DWMRI) is an imaging technique that is sensitive to the Brownian motion of water in tissues [1,2]. Quantitative information can be obtained, and this information is usually displayed as maps of a parameter called the apparent diffusion coefficient (ADC). A high ADC indicates relatively free Brownian motion of water, whereas a low ADC is indicative of restricted motion [2]. The Brownian motion of water is restricted by organelles, cell membranes, and extracellular fibers, and consequently,

\footnotetext{
* Correspondence: einar.k.rofstad@rr-research.no

Group of Radiation Biology and Tumor Physiology, Department of Radiation Biology, Institute for Cancer Research, Oslo University Hospital, Nydalen, Box 4953, Oslo N-0424, Norway
}

ADC maps can provide information on the microstructure and composition of tissues. For example, studies of tumors have shown that high cell density gives rise to low ADC values, whereas high ADC values are measured in necrotic tissue [1,2].

DW-MRI has several potentially useful applications in the oncology and is emerging as a powerful tool in rently being used to distinguish malignant from benign lesions, to differentiate post-therapeutic changes from residual tumor tissue, to detect recurrent tumors, lymph node involvement, and distant metastases, and to predict and monitor response to treatment [3-5]. DW-MRI appears to be particularly promising for detecting metastatic disease, both in regional lymph nodes and distant 
organs [3]. Furthermore, several studies have suggested that ADC may increase rapidly during and after radiation therapy in radiosensitive tumors, an effect that has been attributed to increased water diffusion due to cell death, development of edema, and increased microvessel leakiness $[4,5]$. However, the prognostic value of the pretreatment ADC map and its potential as a biomarker of outcome of treatment are unclear. On the one hand, low pretreatment ADC values have been shown to correlate with several established histological and clinical biomarkers of poor prognosis [6,7]. On the other hand, several studies have suggested that high pretreatment ADC values are indicative of poor response to treatment, possibly because of an association between tumor hypoxia and necrosis $[8,9]$.

Detailed studies of associations between pretreatment $\mathrm{ADC}$ values and the microenvironment of tumors are needed to evaluate the usefulness of DW-MRI as a tool for providing biomarkers of tumor aggressiveness and treatment outcome. Several parameters of the tumor microenvironment including the level of hypoxia and interstitial hypertension have been shown to be associated with metastatic growth, treatment failure, and poor disease-free and overall survival rates [10-12]. In the study reported here, human melanoma xenografts were used to investigate whether DW-MRI may provide information on these microenvironmental parameters and the microenvironment-associated metastatic propensity of tumors. The A-07 and R-18 melanoma lines were selected for the study because the tumors of these lines differ substantially in cell density and do not show necrotic regions [13].

\section{Methods}

\section{Tumor models}

A-07 and R-18 human melanoma xenografts growing in adult female BALB/c nu/nu mice were used as tumor models [13]. Tumors were initiated from cells cultured in RPMI-1640 (25 mmol/L HEPES and L-glutamine) medium supplemented with $13 \%$ bovine calf serum, $250 \mathrm{mg} / \mathrm{L}$ penicillin, and $50 \mathrm{mg} / \mathrm{L}$ streptomycin. Approximately $3.5 \times 10^{5}$ cells in $10 \mu \mathrm{L}$ of Hanks' balanced salt solution were inoculated intradermally in the hind leg. After the cell inoculation, the mice were monitored daily for tumor growth. Experiments were started when the tumors had grown to a volume of $\sim 400 \mathrm{~mm}^{3}$. DWMRI and IFP measurements were carried out with mice anesthetized with fentanyl citrate $(0.63 \mathrm{mg} / \mathrm{kg})$, fluanisone $(20 \mathrm{mg} / \mathrm{kg})$, and midazolam $(10 \mathrm{mg} / \mathrm{kg})$. Animal care and experimental procedures were in concordance with the U.S. Public Health Service Policy on Humane Care and Use of Laboratory Animals as well as Norwegian legislation and were approved by the Norwegian National Animal Research Authority.

\section{DW-MRI}

DW-MRI was carried out with a 1.5-T whole-body clinical scanner (Signa; General Electric, Milwaukee, WI) and a slotted tube resonator transceiver coil constructed for mice. The coil was insulated with styrofoam to prevent excessive heat loss from the animals. The body core temperature of the mice was kept at $37-38^{\circ} \mathrm{C}$ during imaging by using a thermostatically regulated heating pad. The tumors were positioned in the isocenter of the magnet and were imaged axially in a single section through the tumor center by applying a diffusion-weighted singleshot fast spin echo sequence with $\mathrm{ETL}=84$ and $\mathrm{TR}=$ $5002 \mathrm{~ms}$. Images were recorded at a spatial resolution of $0.39 \times 0.39 \times 2.0 \mathrm{~mm}^{3}$ by using an image matrix of $256 \times 256$, a field of view of $10 \times 10 \mathrm{~cm}$, and 10 excitations. Diffusion sensitization gradients were applied in six noncollinear directions with the following $\mathrm{x}, \mathrm{y}$, and $\mathrm{z}$

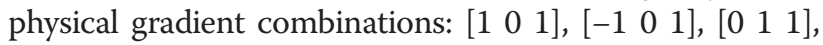
$\left[\begin{array}{lll}0 & 1 & -1\end{array}\right],\left[\begin{array}{lll}1 & 1 & 0\end{array}\right],\left[\begin{array}{lll}-1 & 1 & 0\end{array}\right]$. Five different diffusionweightings with diffusion encoding constants of $b=200$, 400, 600, 800, and $1000 \mathrm{~s} / \mathrm{mm}^{2}$ and corresponding echo times of $\mathrm{TE}=85.0,95.5,103.0,108.9$, and $113.9 \mathrm{~ms}$ were used. The diffusion weightings were achieved by using gradient durations of $\delta=11.6,16.7,20.8,24.3$, and $27.5 \mathrm{~ms}$, a gradient interval of $\Delta=51 \mathrm{~ms}$, and a gradient strength of $G_{\mathrm{D}}=21 \mathrm{mT} / \mathrm{m}$. An image without diffusion weighting $(b=0)$ was recorded for each TE value to compensate for the different TE values associated with the different $b$ values. The $b=0$ images were not included in the calculation of ADC. The total scan time was $\sim 27 \mathrm{~min}$.

ADC maps were produced with in-house-made software developed in Matlab. Briefly, the directional diffusion images were averaged on a voxel-by-voxel basis to non-directional diffusion images. ADC values were calculated for each voxel by fitting the mono-exponential model equation to five-point plots of signal intensity $(S)$ versus $b$ :

$$
\log \left(\frac{S(b, T E)}{S(b=0, T E)}\right)=-b \cdot A D C+c
$$

by using a linear least square fit algorithm. A monoexponential model and $b$ values from 200 to $1000 \mathrm{~s} / \mathrm{mm}^{2}$ were chosen to avoid confounding effects of perfusion, as recommended by Padhani et al. [14]. Parametric images of ADC and the correlation coefficients of the curve fits were generated with the SigmaPlot software. It was verified that the mono-exponential model gave curve fits with correlation coefficients $\geq 0.98$ in more than $95 \%$ of the voxels in all tumors. Median ADC, calculated from the ADC values of the individual voxels, was used as a parameter for the ADC of a tumor. 


\section{Cell density}

Tumor cell density was determined by stereological analysis of histological sections prepared from tissue fixed in phosphate-buffered $4 \%$ paraformaldehyde. The sections were stained with hematoxylin to render cell nuclei clearly visible. The density of cell nuclei was measured by using the method of Brammer and Jung [15], and the density of cells was assumed to be equal to the density of nuclei. Five fields of view, each corresponding to $500-600$ cells, were analyzed for each tumor. Further details of the procedure have been reported elsewhere [16].

\section{Hypoxia}

Pimonidazole [1-[(2-hydroxy-3-piperidinyl)-propyl]-2-nitroimidazole] was administered intraperitoneally in a dose of $30 \mathrm{mg} / \mathrm{kg}$ and used as a marker of tumor hypoxia [17]. The tumors were fixed in phosphate-buffered $4 \%$ paraformaldehyde $\sim 4 \mathrm{~h}$ after the pimonidazole administration. Histological sections were immunostained for hypoxia by using a peroxidase-based indirect staining method [17]. An antipimonidazole rabbit polyclonal antibody (gift from Professor Raleigh, University of North Carolina) was used as primary antibody. Diaminobenzidine was used as chromogen, and hematoxylin was used for counterstaining. Three cross-sections were examined for each tumor. The area of the tumor tissue staining positive for pimonidazole was identified by image analysis [17]. Hypoxic fraction $\left(\mathrm{HF}_{\text {Pim }}\right)$ was calculated as pimonidazole-positive area divided by total tissue area.

\section{Interstitial fluid pressure}

IFP was measured in the tumor center with a Millar SPC 320 catheter equipped with a $2 \mathrm{~F}$ Mikro-Tip transducer (Millar Instruments, Houston, TX) [18]. The catheter was connected to a computer via a Millar TC-510 control unit and a preamplifier. Data acquisition was carried out by using the LabVIEW software (National Instruments, Austin, TX).

\section{Pulmonary metastases}

Microscopic pulmonary metastases were detected by histological examination. Resected lungs were fixed in phosphate-buffered 4\% paraformaldehyde and embedded in paraffin. Histological sections were cut from each lobe at $100-\mu \mathrm{m}$ intervals and stained with hematoxylin and eosin. Groups of five or more tumor cells were scored as a metastasis.

\section{Experimental design}

Three individual experimental series were carried out one subsequent to the other. In the first series, possible associations between ADC and tumor cell density were investigated. In the second series, we investigated whether $\mathrm{HF}_{\mathrm{Pim}}$ and/or IFP may differ between tumors with low and tumors with high cell densities. Possible associations between ADC on the one hand and $\mathrm{HF}_{\mathrm{Pim}}$, IFP, and metastatic propensity on the other were investigated in the third experimental series. The mice were first subjected to DW-MRI, then tumor IFP was measured while the mice were still under anesthesia, and then the mice were euthanized and the tumors and lungs were resected and prepared for assessment of $\mathrm{HF}_{\mathrm{Pim}}$ and metastatic status, respectively.

\section{Statistical analysis}

Experimental data are presented as mean \pm SE unless otherwise stated. Curves were fitted to data by regression analysis. Correlations between variables were searched for by using the Pearson product moment correlation test. Statistical comparisons of data were carried out by using the Student's $t$ test when the data complied with the conditions of normality and equal variance. Under other conditions, comparisons were carried out by non-parametric analysis using the Mann-Whitney rank sum test. The Kolmogorov-Smirnov method was used to test for normality. Probability values $(P)$ and correlation coefficients $\left(R^{2}\right)$ were calculated by using the SigmaStat statistical software. A significance criterion of $P<0.05$ was used.

\section{Results}

Altogether, ADC maps were generated for 35 A- 07 and 36 R-18 tumors, IFP and $\mathrm{HF}_{\text {Pim }}$ were measured in $39 \mathrm{~A}-07$ and 38 R-18 tumors, and cell density was determined for 24. A-07 and 22 R-18 tumors. The A-07 tumors showed a median ADC of $1.0 \times 10^{-3} \mathrm{~mm}^{2} / \mathrm{s}$, a median IFP of $12 \mathrm{mmHg}$, a median $\mathrm{HF}_{\text {Pim }}$ of $3.7 \%$, and a median cell density of $1.8 \times 10^{5}$ cells $/ \mathrm{mm}^{3}$. The corresponding values for the R-18 tumors were $5.3 \times 10^{-4} \mathrm{~mm}^{2} / \mathrm{s}$ (ADC), $28 \mathrm{mmHg}$ (IFP), $5.8 \%\left(\mathrm{HF}_{\text {Pim }}\right)$, and $4.2 \times 10^{5}$ cells $/ \mathrm{mm}^{3}$ (cell density).

ADC was considerably higher in the A- 07 than in the R18 tumors. This is exemplified in Figure 1, which shows the ADC map and the corresponding ADC frequency distribution of a representative A-07 tumor (Figure 1A) and a representative R-18 tumor (Figure 1B). The difference in ADC was most likely a consequence of the lower cell density in the A-07 than in the R-18 tumors. Figure $1 \mathrm{C}$ shows representative images of the histology of an A-07 (left) and an R-18 (right) tumor, illustrating the difference in cell density.

Quantitative studies of possible associations between ADC and cell density were carried out in 10 tumors of each line. Compared with the R-18 tumors, the A-07 tumors showed higher ADC values $(P<0.00001$, Figure $2 \mathrm{~A})$ and lower cell densities $(P<0.00001$, Figure $2 \mathrm{~B})$. The tumors with cell densities below the median value had higher ADC values than the tumors with cell densities above the median value $[P=0.0016$, Figure $2 \mathrm{C}(\mathrm{A}-07) ; P=$ 0.013 , Figure $2 \mathrm{D}(\mathrm{R}-18)]$. The inverse relationship between 

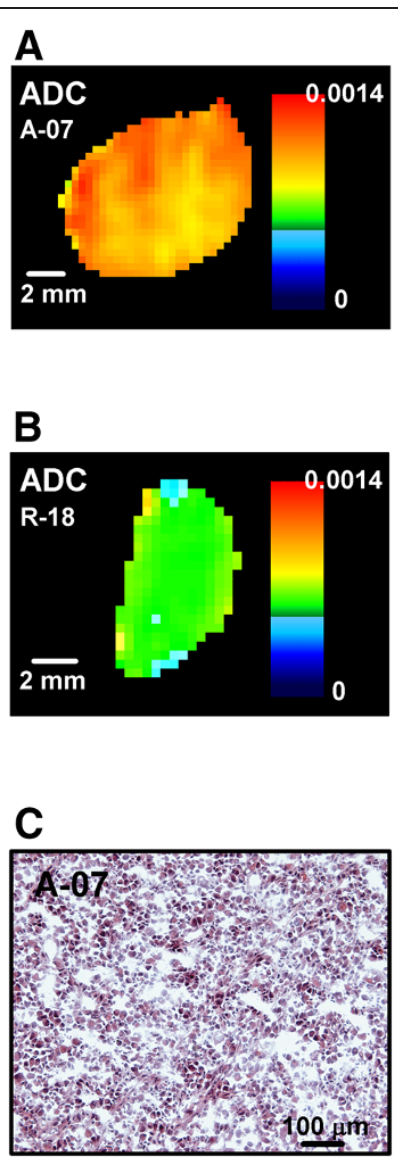

Figure 1 DW-MRI and histological data showing that A-07 tumors had higher ADC values and lower cell densities than R-18 tumors. The ADC map and the corresponding ADC frequency distribution of a representative A-07 tumor (A) and a representative R-18 tumor (B) and the histological appearance of a representative A-07 and a representative R-18 tumor (C).
ADC and cell density was statistically significant at the single tumor level, both within each melanoma line $[P=$ 0.00086 (A-07); $P=0.0023$ (R-18)] and across the two melanoma lines $(P<0.00001)$.

Fourteen A-07 and 12 R-18 tumors were subjected to measurement of IFP and subsequent histological examinations to investigate whether $\mathrm{HF}_{\text {Pim }}$ and/or IFP may differ between tumors with low and tumors with high cell densities. Compared with the tumors with cell densities below the median value, the tumors with cell densities above the median value had both higher $\operatorname{HF}_{\text {Pim }}[P=0.029$, Figure $3 \mathrm{~A}$ (A-07); $P=0.039$, Figure $3 \mathrm{~B}(\mathrm{R}-18)]$ and higher IFP $[P=$ 0.015 , Figure $3 \mathrm{C}$ (A-07); $P=0.042$, Figure 3D (R-18)].

To search for associations between ADC on the one hand and $\mathrm{HF}_{\mathrm{Pim}}$, IFP, and metastatic propensity on the other, 25 A-07 tumors and 26 R-18 tumors were subjected to DW-MRI and measurement of IFP before the host mice were euthanized and the tumors and lungs were resected and prepared for histological examinations. Histological sections of representative tumors stained for hypoxia are presented in Figure 4A (A-07) and Figure 4B (R-18). $\mathrm{HF}_{\text {Pim }}$ differed among the individual tumors from 0 to $12 \%$ (A-07) and from 1 to $23 \%$ (R-18). ADC decreased with increasing $\mathrm{HF}_{\mathrm{Pim}}$, both in the A-07 $(P=0.0080$, Figure $4 \mathrm{C})$ and the $\mathrm{R}-18(P=0.00003$, Figure $4 \mathrm{D})$ tumors. IFP differed among the individual tumors from 5 to $23 \mathrm{mmHg}$ (A-07) and from 12 to $35 \mathrm{mmHg}$ (R-18). ADC decreased significantly with increasing IFP in the A-07 tumors $(P=0.00015$, Figure $4 \mathrm{E})$, but not in the R-18 tumors $(P=0.10$, Figure $4 \mathrm{~F})$. Furthermore, by analyzing the A-07 and R-18 data together, it was shown that there was an inverse correlation between ADC and $\mathrm{HF}_{\text {Pim }}$ and between ADC and IFP across the two melanoma lines $\left[P=0.00009\left(\mathrm{HF}_{\mathrm{Pim}}\right) ; P<0.00001\right.$ (IFP)].

Pulmonary micrometastases were detected in 9 of the 25 mice bearing A-07 tumors, whereas the remaining 16 mice were metastasis-negative. Compared with the tumors of the metastasis-negative mice, the tumors of the metastasis-positive mice had lower ADC values $(P=$ 0.00017, Figure 5A), higher $\operatorname{HF}_{\text {Pim }}(P=0.017$, Figure 5B), 

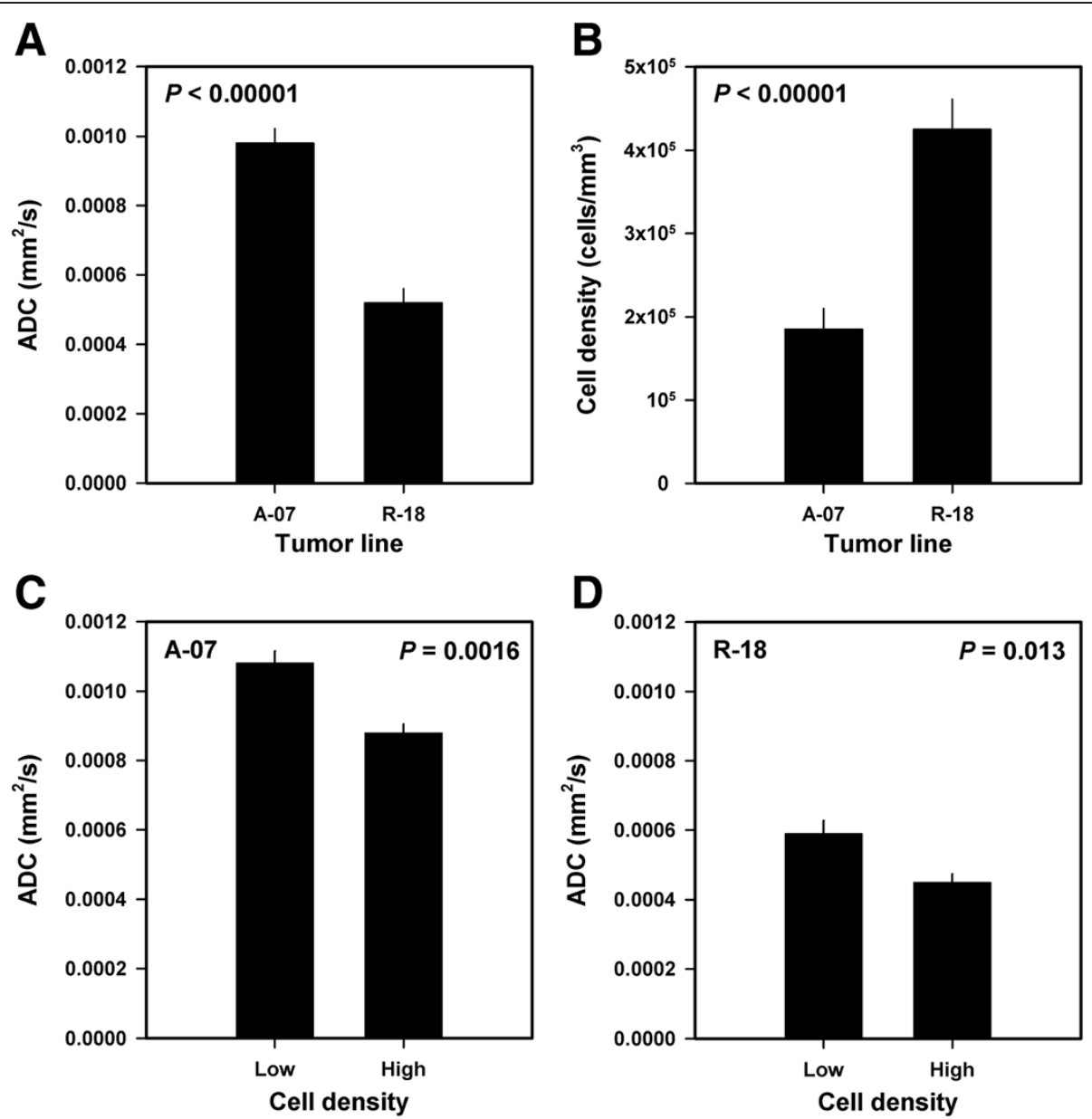

Figure 2 DW-MRI and histological data for A-07 and R-18 melanoma xenografts showing that high ADC was associated with low cell density. Median ADC (A) and cell density (B) for A-07 and R-18 tumors, median ADC for A-07 tumors with low and high cell densities (cell densities below and above the median value of $1.8 \times 10^{5}$ cells $/ \mathrm{mm}^{3}$ ) $\mathbf{( C )}$, and median ADC for R-18 tumors with low and high cell densities (cell densities below and above the median value of $4.2 \times 10^{5}$ cells $/ \mathrm{mm}^{3}$ ) (D). Columns and bars represent mean values and standard errors $[\mathrm{n}=10(\mathbf{A}$ and $\mathbf{B}), n=5$ (C and D)].

and higher IFP $(P=0.00023$, Figure $5 C)$. Metastatic deposits were not detected in any of the mice bearing R-18 tumors.

\section{Discussion}

The possibility that the pretreatment ADC map may provide a useful biomarker of the outcome of cancer has generated a large number of clinical investigations involving several tumor types [3-8]. Some studies suggest that poor clinical outcome is associated with low $\mathrm{ADC}$ values, whereas high ADC values have been found to be associated with poor outcome in other studies $[3,5,9,19]$. The reasons for these apparently conflicting observations have not been identified, possibly because DW-MRI has not been combined with detailed studies of biological and physiological characteristics of the imaged tissue.
In the present preclinical study, tumors subjected to DW-MRI were examined with respect to cell density, fraction of hypoxic tissue, level of interstitial hypertension, and metastatic status. Tumors with low ADC values were found to have high cell densities, consistent with the general theory that low ADC indicates restricted Brownian motion of water [1-3]. Furthermore, low ADC values in tumors were associated with high $\mathrm{HF}_{\mathrm{Pim}}$, high IFP, and elevated metastatic propensity in A-07 tumors and with high $\mathrm{HF}_{\text {Pim }}$ in $\mathrm{R}-18$ tumors.

Tumor hypoxia is a result of an imbalance between the rate of oxygen supply and the rate of oxygen consumption [20]. The rate of oxygen supply is determined primarily by the vascular architecture and the rate of blood flow, whereas the rate of oxygen consumption is determined primarily by the respiratory activity of the tumor cells and the cell density. In the A-07 and R-18 

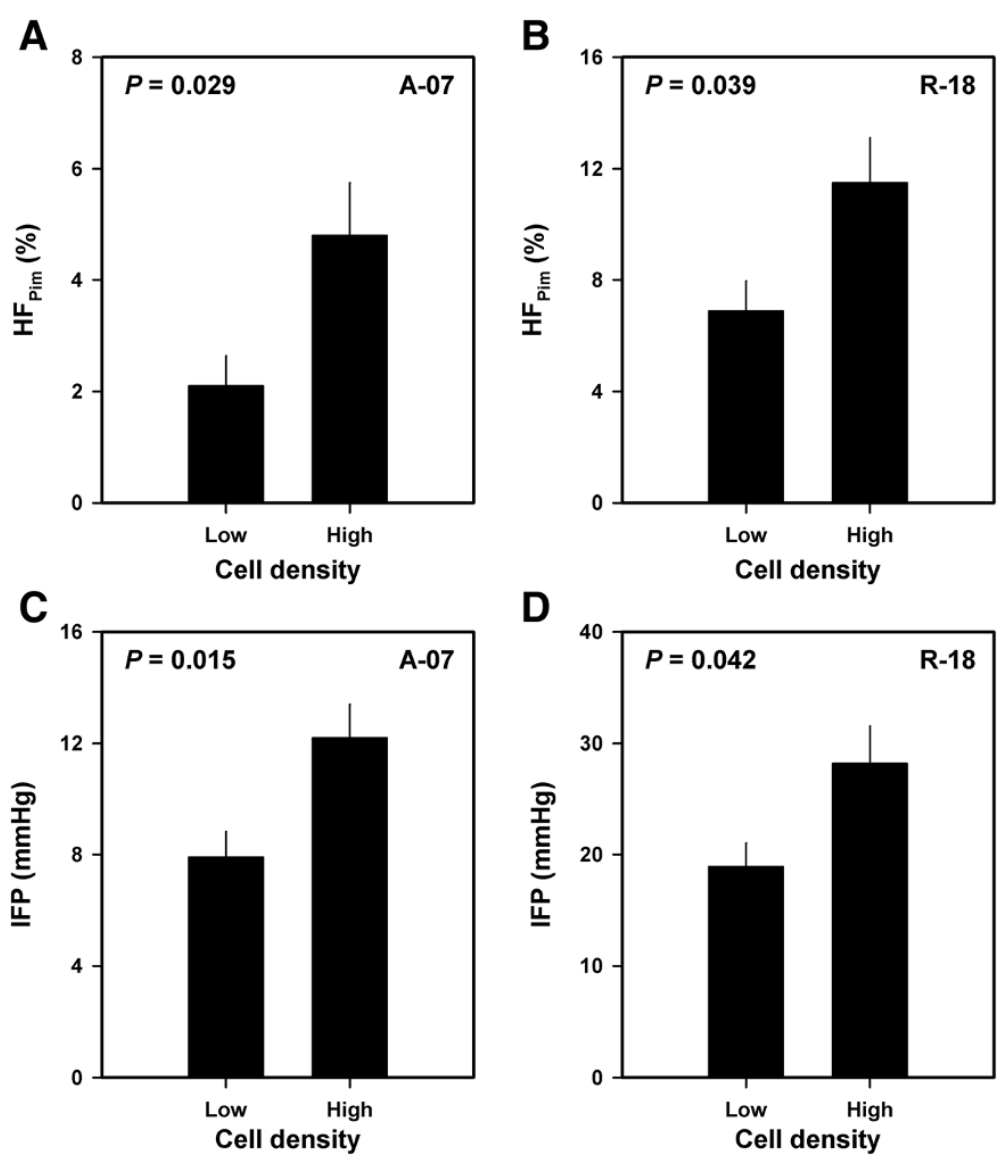

Figure 3 Hypoxia, IFP, and cell density data for A-07 and R-18 melanoma xenografts showing that high $\mathrm{HF}_{\text {pim }}$ and high IFP were associated with high cell density. $\mathrm{HF}_{\text {Pim }}$ for A-07 tumors with low and high cell densities (cell densities below and above the median value of $1.8 \times 10^{5}$ cells $/ \mathrm{mm}^{3}$ ) (A), $\mathrm{HF}_{\text {Pim }}$ for R-18 tumors with low and high cell densities (cell densities below and above the median value of $4.2 \times 10^{5}$ cells $/ \mathrm{mm}^{3}$ ) (B), IFP for A-07 tumors with low and high cell densities (C), and IFP for R-18 tumors with low and high cell densities (D). Columns and bars represent mean values and standard errors ( $n=7$ for A- 07 and $n=6$ for R-18).

lines, tumors with high cell densities showed higher $\mathrm{HF}_{\text {Pim }}$ than tumors with low cell densities, suggesting that the intertumor heterogeneity in fraction of hypoxic tissue was influenced significantly by the intertumor heterogeneity in cell density. The inverse correlations between $\mathrm{ADC}$ and $\mathrm{HF}_{\mathrm{Pim}}$ in A-07 and R-18 tumors were therefore most likely an indirect consequence of the relationship between ADC and cell density.

Interstitial hypertension in tumors is primarily a consequence of high resistance to blood flow, low resistance to transcapillary fluid flow, decreased interstitial hydraulic conductivity, and impaired lymphatic drainage [21]. In tumors with highly permeable blood vessels like the A-07 and R-18 tumors, the intertumor heterogeneity in IFP is mainly a result of intertumor heterogeneity in resistance to both blood flow and interstitial fluid flow [22]. High cell density is associated with high resistance to interstitial fluid flow [23], and furthermore, cell division in rapidly growing tumors with high cell densities may cause mechanical pressure on vessel walls, leading to local vessel narrowings and, hence, increased resistance to blood flow [24]. IFP was higher in tumors with high cell densities than in tumors with low cell densities in the A-07 and R-18 lines. The association between ADC and IFP observed here was therefore most likely an indirect consequence of the relationship between ADC and cell density.

There is significant evidence from clinical studies that poor disease-free and overall rates in cancer are associated with extensive hypoxia and interstitial hypertension in the primary tumor [10-12]. Preclinical studies have revealed that pulmonary and lymph node metastasis is promoted by tumor hypoxia $[25,26]$ and highly elevated IFP $[27,28]$. Previous studies have shown that metastasis is associated with hypoxia and the level of interstitial hypertension also in A-07 tumors [28,29]. 
A

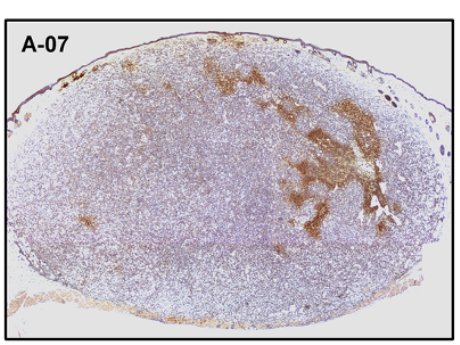

C

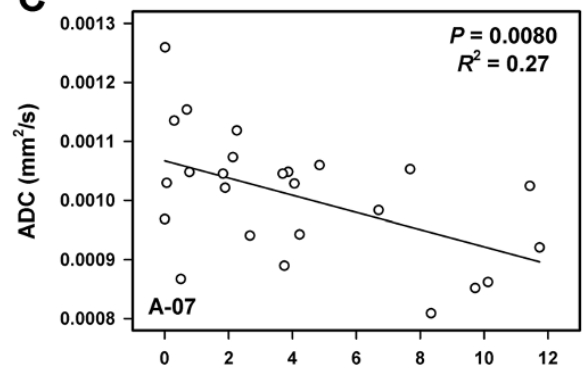

E

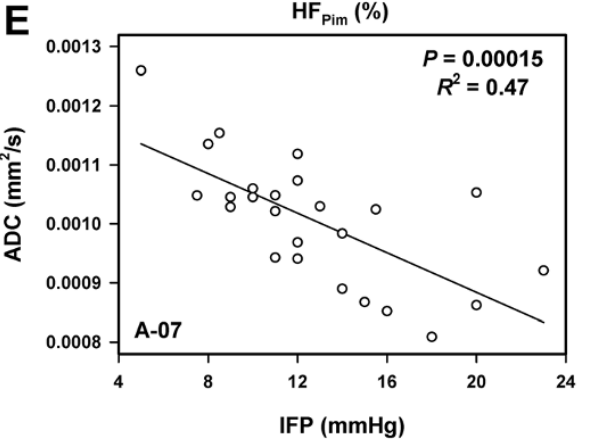

B
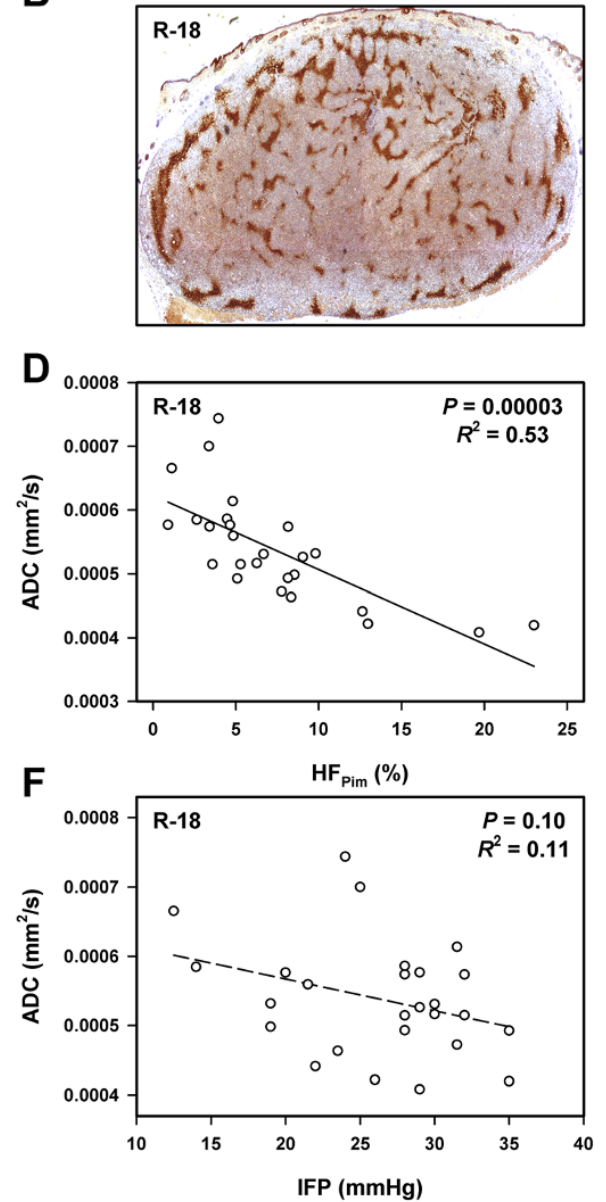

Figure 4 DW-MRI, hypoxia, and IFP data for A-07 and R-18 melanoma xenografts showing that ADC decreased with increasing $\mathrm{HF}_{\mathrm{Pim}}$ and increasing IFP. Histological sections of a representative A-07 tumor (A) and a representative R-18 tumor (B) stained with anti-pimonidazole antibody to visualize hypoxic tissue, plots of median ADC versus $\mathrm{HF}_{\text {Pim }}$ for $\mathrm{A}-07$ (C) and R-18 (D) tumors, and plots of median ADC versus IFP for A-07 (E) and R-18 (F) tumors. Points represent single tumors. Curves were fitted to data by regression analysis.

These observations were confirmed in the present work, which showed that metastatic A-07 tumors had higher $\mathrm{HF}_{\text {Pim }}$ and higher IFP than their nonmetastatic counterparts. The lower ADC in the metastatic than in the nonmetastatic A-07 tumors was most likely an indirect consequence of higher cell densities in the metastatic tumors, since both high $\mathrm{HF}_{\mathrm{Pim}}$ and high IFP were associated with metastasis, high cell density, and low ADC.

Taken together, our data suggest that low rather than high ADC values are indicative of tumor aggressiveness and poor prognosis in cancer. This suggestion is consistent with some clinical investigations, which have shown that low ADC values in untreated tumors may be associated with several established biomarkers of poor prognosis in breast [6], prostate [7], and lung cancer [30]. Recent clinical studies have also provided significant evidence that low ADC values may be a characteristic feature of primary tumors that are likely to metastasize [31]. Moreover, aggressive tumors have been demonstrated to have lower ADC values than indolent tumors in patients with localized prostate cancer [32], and the ADC of the primary tumor has been found to be lower in patients with metastatic disease than in patients without detectable metastases in high-grade urothelial carcinoma of the bladder [33].

In contrast, some preclinical and clinical studies have suggested that poor response to treatment is associated with high pretreatment ADC values [8,9]. High ADC values may be associated with treatment resistance if the treatment resistance is caused by hypoxia and the tumors show significant hypoxia-associated necrosis. Proper evaluation of the prognostic and predictive potential of DW-MRI may require that voxels in necrotic tumor regions are excluded from ADC maps, since ADC 


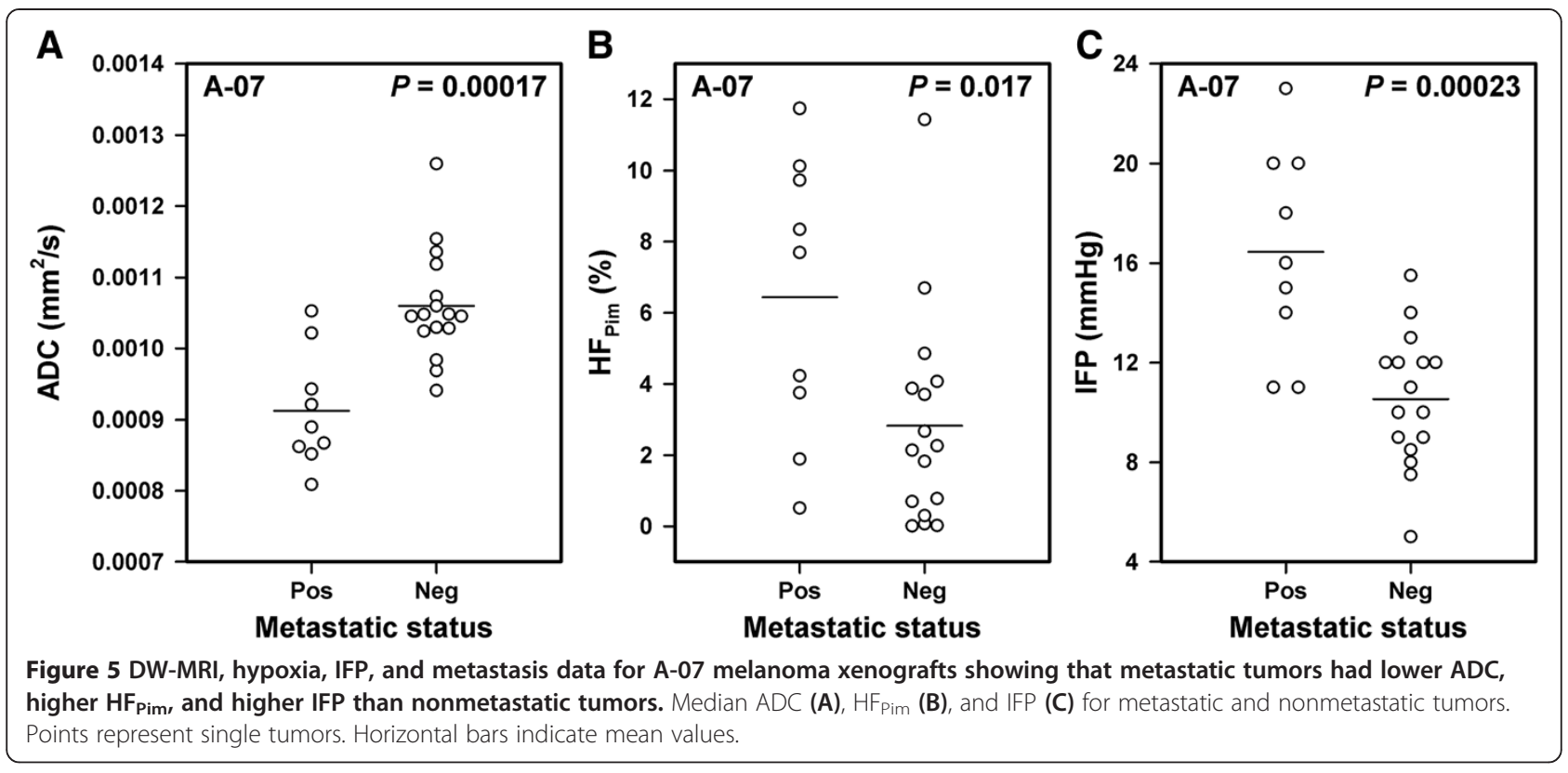

maps may be confounded significantly by the highly elevated $A D C$ values seen in necrotic tissue. Necrotic tumor regions may be identified by combining DW-MRI with contrast-enhanced MRI [34]. It is important to note that A-07 and R-18 tumors in general do not develop necrotic regions until they reach a volume of $700-800 \mathrm{~mm}^{3}$ [13], and necrosis was not seen in any of the histological sections examined in the present work.

In preclinical studies, each tumor line represents a single patient, whereas individual tumors of the same line represent copies of the same tumor. These tumor copies have the same genetic background, but may show substantially different physiological microenvironments owing to the stochastic nature of tumor angiogenesis. By studying many tumors of the same xenograft line, associations between MRI-derived parameters and parameters of the tumor microenvironment can be revealed. However, these MRI-derived parameters cannot be expected to be prognostic for the clinical outcome of cancer unless they show significant correlations with microenvironmental parameters across tumor lines. Interestingly, ADC showed significant inverse correlations with cell density, $\mathrm{HF}_{\mathrm{Pim}}$, and IFP across the two melanoma lines included in this study, consistent with the hypothesis that tumor ADC may be an important prognostic factor in cancer.

The hypothesis that low ADC may indicate poor prognosis is also supported by the observation that low ADC was associated with pulmonary metastasis in A-07 tumors. On the other hand, the observation that R-18 tumors showed lower ADC values than the A-07 tumors and did not give rise to pulmonary metastases is apparently inconsistent with this hypothesis. Previous studies have revealed that the A-07 and R-18 xenograft lines show organ-specific metastatic patterns (i.e., A-07 tumors metastasize primarily to the lungs, whereas R-18 tumors preferentially develop lymph node metastases) [13]. The incidence of lymph node metastases was not scored in the present investigation. However, previous investigations have revealed that the development of lymph node metastases in R-18 tumors is associated with poor blood supply and high fractions of hypoxic tissue [35] and, furthermore, that hypoxia promotes lymph node metastasis in R-18 tumors primarily by up-regulating the expression of the urokinase-type plasminogen activator receptor [36].

\section{Conclusions}

This study confirmed that low ADC is associated with high cell density in tumors and revealed that tumors with high cell densities may have high hypoxic fractions, high IFP, and elevated metastatic propensity. Low ADC was found to be associated with high $\mathrm{HF}_{\mathrm{Pim}}$, high IFP, and metastasis, primarily because these parameters were all associated with high tumor cell density. Taken together, our observations suggest that low pretreatment ADC values may be associated with a hostile microenvironment in tumors. Consequently, the possibility that DW-MRI may provide biomarkers of microenvironment-associated tumor aggressiveness and treatment resistance merits clinical investigations. 


\section{Abbreviations}

ADC: Apparent diffusion coefficient; DW-MRI: Diffusion-weighted magnetic resonance imaging; ETL: Echo train length; $\mathrm{HF}_{\text {Pim }}$ : Hypoxic fraction assessed by using pimonidazole as a hypoxia marker; IFP: Interstitial fluid pressure; TE: Echo time; TR: Repetition time.

\section{Competing interests}

The authors declare that they have no competing interests.

\section{Authors' contributions}

$\mathrm{TH}$ was involved in conceiving the study, designing and performing experiments, analyzing and interpreting data, carrying out statistical analyses, and preparing the manuscript. CE was involved in designing experiments, interpreting data, and preparing the manuscript. KG was involved in performing experiments and analyzing and interpreting data. EKR was involved in conceiving the study, interpreting data, and preparing the manuscript. All authors read and approved the final manuscript.

\section{Acknowledgements}

Financial support was received from the Norwegian Cancer Society and the South-Eastern Norway Regional Health Authority.

Received: 30 September 2013 Accepted: 12 February 2014 Published: 15 February 2014

\section{References}

1. Padhani AR: Diffusion magnetic resonance imaging in cancer patient management. Semin Radiat Oncol 2011, 21:119-140.

2. Yankeelov TE, Arlinghaus LR, Li X, Gore JC: The role of magnetic resonance imaging biomarkers in clinical trials of treatment response in cancer. Semin Oncol 2011, 38:16-25.

3. Bonekamp S, Corona-Villalobos CP, Kamel IR: Oncologic applications of diffusion-weighted MRI in the body. J Magn Reson Imaging 2012, 35:257-279.

4. Li SP, Padhani AR: Tumor response assessments with diffusion and perfusion MRI. J Magn Reson Imaging 2012, 35:745-763.

5. Liu Y, Bai R, Sun H, Liu H, Zhao X, Li Y: Diffusion-weighted imaging in predicting and monitoring the response of uterine cervical cancer to combined chemoradiation. Clin Radiol 2009, 64:1067-1074

6. Razek AA, Gaballa G, Denewer A, Nada N: Invasive ductal carcinoma: correlation of apparent diffusion coefficient value with pathological prognostic factors. NMR Biomed 2010, 23:619-623.

7. Turkbey B, Shah VP, Pang Y, Bernardo M, Xu S, Kruecker J, Locklin J, Baccala AA, Rastinehad AR, Merino MJ, Shih JH, Wood BJ, Pinto PA, Choyke PL: Is apparent diffusion coefficient associated with clinical risk scores for prostate cancers that are visible on 3-T MR images? Radiology 2011, 258:488-495.

8. Roth Y, Tichler T, Kostenig G, Ruiz-Cabello J, Maier SE, Cohen JS, Orenstein A, Mardor $Y$ : High-b-value diffusion-weighted MR imaging for pretreatment prediction and early monitoring of tumor response to therapy in mice. Radiology 2004, 232:685-692.

9. Sun YS, Zhang XP, Tang L, Ji JF, Gu J, Cai Y, Zhang XY: Locally advanced rectal carcinoma treated with preoperative chemotherapy and radiation therapy: preliminary analysis of diffusion-weighted MR imaging for early detection of tumor histopathologic downstaging. Radiology 2010, 254:170-178.

10. Nordsmark M, Bentzen SM, Rudat V, Brizel D, Lartigau E, Stadler P, Becker A Adam M, Molls M, Dunst J, Terris DJ, Overgaard J: Prognostic value of tumor oxygenation in 397 head and neck tumors after primary radiation therapy. An international multi-center study. Radiother Oncol 2005, 77:18-24.

11. Fyles A, Milosevic M, Pintilie M, Syed A, Levin W, Manchul L, Hill RP: Long-term performance of interstitial fluid pressure and hypoxia as prognostic factors in cervix cancer. Radiother Oncol 2006, 80:132-137.

12. Vaupel $P$, Mayer A: Hypoxia in cancer: significance and impact on clinical outcome. Cancer Metastasis Rev 2007, 26:225-239.

13. Rofstad EK: Orthotopic human melanoma xenograft model systems for studies of tumour angiogenesis, pathophysiology, treatment sensitivity and metastatic pattern. Br J Cancer 1994, 70:804-812.

14. Padhani AR, Liu G, Koh DM, Chenevert TL, Thoeny HC, Takahara T, DzikJurasz A, Ross BD, Van Cauteren M, Collins D, Hammoud DA, Rustin GJ, Taouli B, Choyke PL: Diffusion-weighted magnetic resonance imaging as a cancer biomarker: consensus and recommendations. Neoplasia 2009 2:102-125.
15. Brammer I, Jung H: Morphometry of irradiated tumors. In Rodent Tumor Models in Experimental Cancer Therapy. Edited by Kallman RF. Oxford: Pergamon Press; 1987:97-100.

16. Egeland TAM, Simonsen TG, Gaustad JV, Gulliksrud K, Ellingsen C, Rofstad EK: Dynamic contrast-enhanced magnetic resonance imaging of tumors: preclinical validation of parametric images. Radiat Res 2009, 172:339-347.

17. Rofstad EK, Måseide K: Radiobiological and immunohistochemical assessment of hypoxia in human melanoma xenografts: acute and chronic hypoxia in individual tumors. Int J Radiat Biol 1999, 75:1377-1393.

18. Ozerdem $U$, Hargens AR: A simple method for measuring interstitial fluid pressure in cancer tissues. Microvasc Res 2005, 70:116-120.

19. Padhani AR, Koh DM, Collins DJ: Whole-body diffusion-weighted MR imaging in cancer: current status and research directions. Radiology 2011, 261:700-718.

20. Gulledge CJ, Dewhirst MW: Tumor oxygenation: a matter of supply and demand. Anticancer Res 1996, 16:741-750.

21. Heldin $\mathrm{CH}$, Rubin K, Pietras K, Östman A: High interstitial fluid pressure-an obstacle in cancer therapy. Nat Rev Cancer 2004, 4:806-813.

22. Lunt SJ, Fyles A, Hill RP, Milosevic M: Interstitial fluid pressure in tumors: therapeutic barrier and biomarker of angiogenesis. Future Oncol 2008, 4:793-802.

23. Milosevic M, Fyles A, Hill RP: The relationship between elevated interstitial fluid pressure and blood flow in tumors: a bioengineering analysis. Int J Radiat Oncol Biol Phys 1999, 43:1111-1123.

24. Padera TP, Stoll BR, Tooredman JB, Capen D, di Tomaso E, Jain RK: Pathology: cancer cells compress intratumour vessels. Nature 2004, 427:695.

25. Cairns RA, Kalliomäki T, Hill RP: Acute (cyclic) hypoxia enhances spontaneous metastasis of KHT murine tumors. Cancer Res 2001, 61:8903-8908.

26. Rofstad EK, Galappathi K, Mathiesen B, Ruud EBM: Fluctuating and diffusion-limited hypoxia in hypoxia-induced metastasis. Clin Cancer Res 2007, 13:1971-1978.

27. Hompland T, Ellingsen C, Øvrebø KM, Rofstad EK: Interstitial fluid pressure and associated lymph node metastasis revealed in tumors by dynamic contrast-enhanced MRI. Cancer Res 2012, 72:4899-4908.

28. Rofstad EK, Tunheim SH, Mathiesen B, Graff BA, Halsør EF, Nilsen K, Galappathi K: Pulmonary and lymph node metastasis is associated with primary tumor interstitial fluid pressure in human melanoma xenografts. Cancer Res 2002, 62:661-664.

29. Rofstad EK, Gaustad JV, Egeland TAM, Mathiesen B, Galappahi K: Tumors exposed to acute cyclic hypoxic stress show enhanced angiogenesis, perfusion and metastatic dissemination. Int J Cancer 2010, 127:1535-1546.

30. Razek AA, Fathy A, Gawad TA: Correlation of apparent diffusion coefficient value with prognostic parameters of lung cancer. J Comput Assist Tomogr 2011, 35:248-252.

31. Dietzel M, Baltzer PA, Vag T, Gröschel T, Gajda M, Camara O, Kaiser WA: Application of breast MRI for prediction of lymph node metastases systematic approach using 17 individual descriptors and a dedicated decision tree. Acta Radiol 2010, 51:885-894.

32. de Souza NM, Riches SF, Vanas NJ, Morgan VA, Ashley SA, Fisher C, Payne GS, Parker C: Diffusion-weighted magnetic resonance imaging: a potential non-invasive marker of tumour aggressiveness in localized prostate cancer. Clin Radiol 2008, 63:774-782.

33. Rosenkrantz AB, Mussi TC, Spieler B, Melamed J, Taneja SS, Huang WC High-grade bladder cancer: associations of the apparent diffusion coefficient with metastatic disease: preliminary results. J Magn Reson Imaging 2012, 35:1478-1483.

34. Egeland TAM, Gaustad JV, Galappathi K, Rofstad EK: Magnetic resonance imaging of tumor necrosis. Acta Oncol 2011, 50:427-434.

35. Øvrebø KM, Gulliksrud K, Mathiesen B, Rofstad EK: Assessment of tumor radioresponsiveness and metastatic potential by dynamic contrastenhanced magnetic resonance imaging. Int J Radiat Oncol Biol Phys 2011, 81:255-261

36. Rofstad EK, Rasmussen H, Galappathi K, Mathiesen B, Nilsen K, Graff BA Hypoxia promotes lymph node metastasis in human melanoma xenografts by up-regulating the urokinase-type plasminogen activator receptor. Cancer Res 2002, 62:1847-1853.

doi:10.1186/1471-2407-14-92

Cite this article as: Hompland et al.: DW-MRI in assessment of the hypoxic fraction, interstitial fluid pressure, and metastatic propensity of melanoma xenografts. BMC Cancer 2014 14:92. 